\title{
Article \\ Synthetic Weyl Points of the Shear Horizontal Guided Waves in One-Dimensional Phononic Crystal Plates
}

\author{
Hongbo Zhang ${ }^{1,2}$, Shaobo Zhang ${ }^{1}$, Jiang Liu ${ }^{1, *}$ and Bilong Liu ${ }^{1}$ \\ 1 School of Mechanical \& Automobile Engineering, Qingdao University of Technology, \\ No. 777 Jialingjiang Road, Qingdao 266520, China; zhanghongbo@qtech.edu.cn (H.Z.); \\ zhangshaobo@qut.edu.cn (S.Z.); liubilong@qtech.edu.cn (B.L.) \\ 2 Key Lab of Industrial Fluid Energy Conservation and Pollution Control, Ministry of Education, \\ Qingdao University of Technology, No. 777 Jialingjiang Road, Qingdao 266520, China \\ * Correspondence: liujiang@qtech.edu.cn; Tel.: +86-131-6502-8316
}

check for updates

Citation: Zhang, H.; Zhang, S.; Liu, J.; Liu, B. Synthetic Weyl Points of the Shear Horizontal Guided Waves in One-Dimensional Phononic Crystal Plates. Appl. Sci. 2022, 12, 167. https://doi.org/10.3390/ app12010167

Academic Editors: Baizhan Xia, Qingbo He, Tianzhi Yang, Tianxi Jiang and Vincent Laude

Received: 4 November 2021 Accepted: 19 December 2021 Published: 24 December 2021

Publisher's Note: MDPI stays neutral with regard to jurisdictional claims in published maps and institutional affiliations.

Copyright: () 2021 by the authors Licensee MDPI, Basel, Switzerland. This article is an open access article distributed under the terms and conditions of the Creative Commons Attribution (CC BY) license (https:// creativecommons.org/licenses/by/ $4.0 /)$.

\begin{abstract}
Weyl physics in acoustic and elastic systems has drawn extensive attention. In this paper, Weyl points of shear horizontal guided waves are realized by one-dimensional phononic crystal plates, in which one physical dimension plus two geometrical parameters constitute a synthetic threedimensional space. Based on the finite element method, we have not only observed the synthetic Weyl points but also explored the Weyl interface states and the reflection phase vortices, which have further proved the topological phase interface states. As the first realization of three-dimensional topological phases through one-dimensional phononic crystal plates in the synthetic dimension, this research demonstrates the great potential of applicable one-dimensional plate structural systems in detecting higher-dimensional topological phenomena.
\end{abstract}

Keywords: one-dimensional phononic crystal plate; shear horizontal guided wave; synthetic dimension; Weyl point

\section{Introduction}

Weyl semimetals, which have linear intersections at the isolated momentum in threedimensional (3D) materials, have attracted great research interests [1-3]. The linear intersection points named as Weyl points (WPs) are stable in the face of small disturbances [4-6]. Weyl semimetals exhibit various intriguing features, such as chiral anomalies and topological Femi interface states [5-9].

Researcher has introduced Weyl physics into sonic crystals [10-14], and the related topological surface states have also been extensively explored. Topological interface states usually occur at two insulator interfaces with different topological phases. Besides acoustic topological states, the fields of electromagnetic [15,16], plasmas [17], and elastic waves [18-21] have been investigated the topological states. However, the vast majority of Weyl crystals have a real 3D structure, which are complicated in manufacturing samples and detecting signals. The development of synthetic dimensions has been used to alleviate the problems encountered in real 3D space [22,23]. There are many ways to create synthetic dimensions, and the development of synthetic dimensions even provides a corresponding way to explore systems beyond 3D systems [24].

Different from previous studies [13,25-27], synthetic dimensions are used to replace purely physical dimension. By constructing two independent structural parameters in Weyl Hamiltonian with wave vector, one-dimensional (1D) phononic crystal (PC) plates are used to explore interesting Weyl physics. Different from the Dirac points, the WPs interacting with other bands, are immune to interference since the wave vector maintains a good quantum number $[28,29]$. The certain topological charge of each WP can be calculated by the Chern number [30]. Although the WPs are usually investigated in real 3D structures, which can also be studied theoretically in the synthetic dimension [31]. 
In this paper, synthetic WPs are observed, the transmission spectra (TS) and the reflection phases (RPs) with changing geometrical parameters are studied. The intrinsic vortex structure of the RP is confirmed, which provides a new representation for the topologically robust WPs. It is proved that the RP of the truncated one-dimensional (1D) phononic crystal (PC) plates shows the vortex structure around the synthetic WPs, which carries the same topological charge as the corresponding WPs. This study may facilitate several operational applications. For example, the interface states under strong constraints can enhance the elastic energy and thus can be combined into directional elastic antenna structures, providing a technical means for communication and detection.

\section{Materials and Methods}

As shown in Figure 1, the 1D PC plate is made of silicon, whose material parameters are as follows: mass density $\rho=2332 \mathrm{~kg} / \mathrm{m}^{3}$, shear wave velocity $v_{\mathrm{t}}=5840.9 \mathrm{~m} / \mathrm{s}$, and elastic constant $C_{44}=79.56 \mathrm{GPa}$. Each unit cell contains four layers, whose characteristic length is $t_{1} \sim t_{4}$, where $t_{1}+t_{3}=2 a / 3$ and $t_{2}+t_{4}=a / 3$. The lattice constant is $a=9 \mathrm{~mm}$, the height of pile $h_{1}=3 \mathrm{~mm}$ and $h_{2}=4.2 \mathrm{~mm}$. The above geometric parameters remain unchanged in the discussion of this paper. Two additional geometrical parameters are defined as $p=\left(t_{1}-t_{3}\right) /\left(t_{1}+t_{3}\right)$ and $q=\left(t_{2}-t_{4}\right) /\left(t_{2}+t_{4}\right)$. Since the values of $t_{1} \sim t_{4}$ are positive, both parameters range from $[-1,1]$, which forms a closed parameter space. A virtual 3D parameter space can be formed by these two parameters plus the wave vector, where WPs can be investigated.

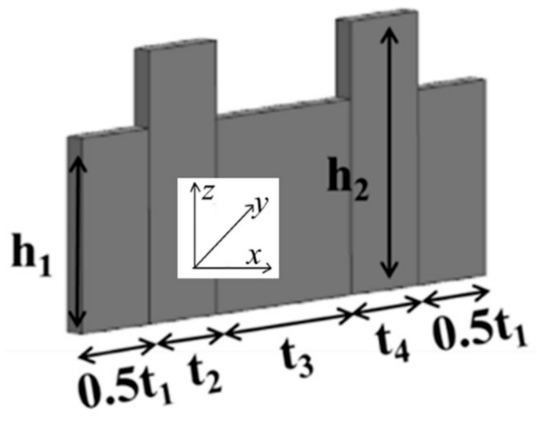

(a)

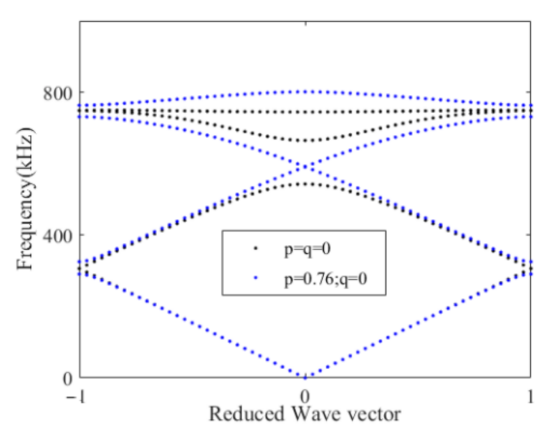

(b)

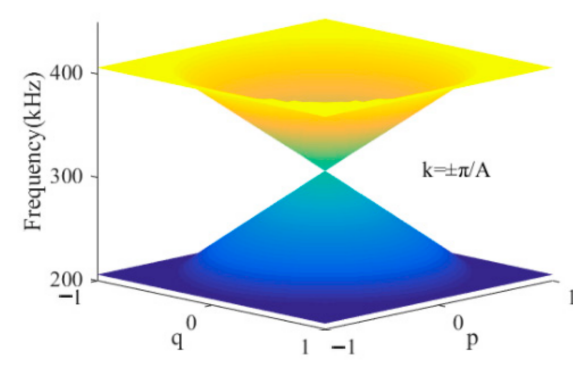

(c)

Figure 1. The synthetic Weyl points in 1D PC plates. (a) The unit cell consists of four layers. (b) Dispersion curves with different $p$ and $q$ values (c) Bulk dispersions plotted for bands 1 and 2 with the wave vector plus two structural parameters.

When the Shear horizontal (SH) guided waves propagate in the 1D PC plates, the governing equation is expressed as:

$$
-\rho \omega^{2} U_{2}=T_{12,1}+\left(C_{44} U_{2,3}\right)_{, 3}
$$

where the subscripts 1,2 , and 3 represent the coordinates $x, y$, and $z, U_{2}$ is the displacement vector along the y-direction, $C_{44}$ is the elastic constant, and $T_{12}$ is the stress tensor component.

Based on the Bloch-Floquet theorem, the displacement in PC plates is given as:

$$
\mathbf{u}(\mathbf{r}, t)=\mathbf{u}_{\mathbf{k}}(\mathbf{r}) e^{i(\mathbf{k} \cdot \mathbf{r}+\omega t)},
$$

where $\mathbf{r}=(x, y, z)$ is the position vector and $\mathbf{k}=\left(k_{x}, k_{y}, k_{z}\right)$ is the Bloch wave vector. Since the 1D PC plates are periodic in the $x$ direction, one unit cell is considered. We substitute 
Equation (2) into (1) and discretize the model with sufficient elements. The eigenvalue equation in the finite element method (FEM) framework is:

$$
\left(\mathbf{K}(\mathbf{k})-\omega(\mathbf{k})^{2} \mathbf{M}\right) \mathbf{u}=0,
$$

in which $\mathbf{K}$ and $\mathbf{M}$ stand for stiffness matrix and mass matrix. The band structures for the 1D PC plates can be obtained by scanning the points of the Brillouin zone.

Using the frequency domain study of COMSOL software 5.6, the TS and the RP can be calculated. For TS, the simulation model is shown in Figure 2. A uniform harmonic shear displacement is applied on the left side of the PC plate and the perfectly matched layers (PMLs) are used to avoid reflection. The energy density spectrum normalized by the flat panel is defined as:

$$
I=\frac{\sqrt{v_{2 x^{2}+v_{2 z^{2}}}}}{\sqrt{v_{1 x^{2}+v_{1 z^{2}}}}}
$$

in which $v_{1 x}$ and $v_{1 z}$ stand for the displacement of the receive point with PC plate, while $v_{2 x}$ and $v_{2 z}$ stand for the displacement of the receive point with flat panel.

\begin{tabular}{|l|r|r|r|c|}
\hline PML & flat panel & PC plate or flat panel & flat panel & PML \\
\hline
\end{tabular}

Figure 2. The simulation model for TS.

For the RP calculation, the left PML layer is replaced with a hard boundary condition. With the same excitation, the RP can also be obtained from the rigid boundary.

\section{Results}

Starting from the special case, the synthesis parameters are selected as $p=q=0$, in other words, $t_{1}=t_{3}$, and $t_{2}=t_{4}$. As shown in Figure $1 b$, this case simply doubles the size of the unit cell and the Brillouin zone is folded. Such band folding results in a linear crossover of the first (third) and second (fourth) along the wave vector direction at $k= \pm \pi / a$. Away from the point ( $p=0$ and $q=0)$, band gaps are formed since the degeneration resulting from the band folding is removed, as exemplified by the SC with ( $p=0.76$ and $q=0)$ (blue dotted lines). It can be inferred from Figure 1a that the increase and decrease of $p$ (or $q$ ) from $p=0$ (or $q=0$ ) is not symmetric in the one-dimensional structure. Therefore, the dispersion relation $\omega(p, q, k)$ is expanded at $p=q=0$, the first-order terms $\Delta p$ and $\Delta q$ will play the dominant role and give linear dispersion in the $p$ and $q$ directions. Based on the above analysis, the intersections at $(0,0, \pm \pi / a)$ are WPs in this synthetic dimension.

The intersection between two adjacent bands can also form at the wave number $k=0$ or $k= \pm \pi / a$ when $p=0$ or $q=0$, as guarded by space-reflection symmetry of the 1D PC plate. Let me give an example, an accidental linear degeneracy can also form at $k=0$ with $p=0.76$ and $q=0$ as shown in Figure $1 \mathrm{~b}$. These degenerate points are also WPs in this synthetic dimension, which are further verified by the conic intersections in the bulk dispersions depicted in $p-q$ space, as shown in Figure 1c.

It is noted that the mirror symmetry of our framework ensures that when a WP exists at $(p, q, k)$, there must be corresponding WPs with the same frequency and the same topological charge at $( \pm p, \pm q, \pm k)$. The main difference between our system and the real Weyl crystals is that the total charge does not vanish because the synthesis parameters are not periodic.

To further confirm the synthesized WPs, the frequency-dependent TS through 1D PC plates with different geometrical parameters are investigated. Here we pay attention to the frequency range around the WP formed by the first and second bands. Figure $3 a-c$ shows 95 independent phononic crystal models (including 19 different $p$ and five different $q$ ) and each model consists of 10 periods. The simulation results shows that when $q$ gradually 
approaches 0 , the bandwidth of the transmission gap decreases and disappears. It shows obvious linear crossover near the expected frequency and structural parameters. It is also worth noting that the band structures with negative $q$ are the same as the band structures with the positive " $q$ ", which leads to the same TS.

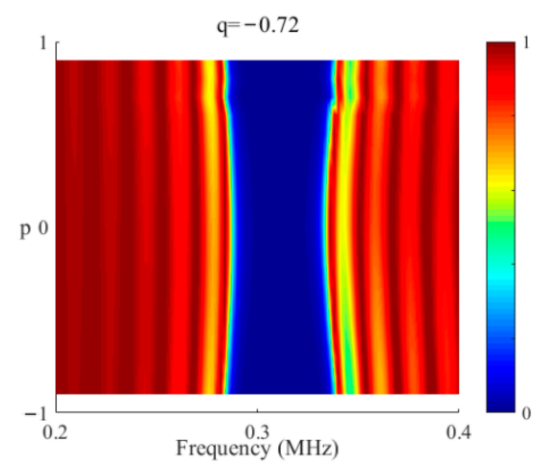

(a)

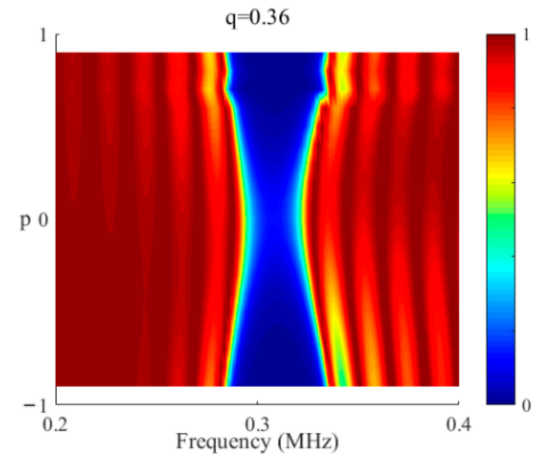

(d)

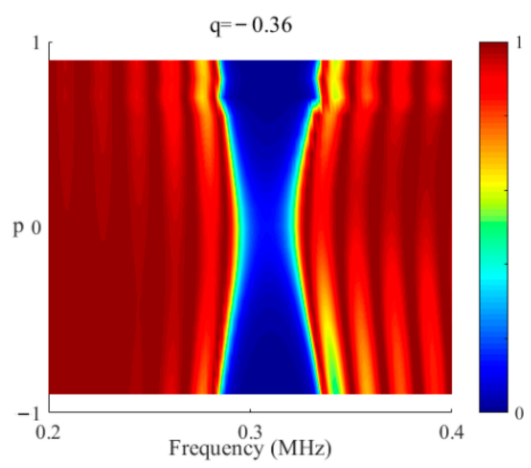

(b)

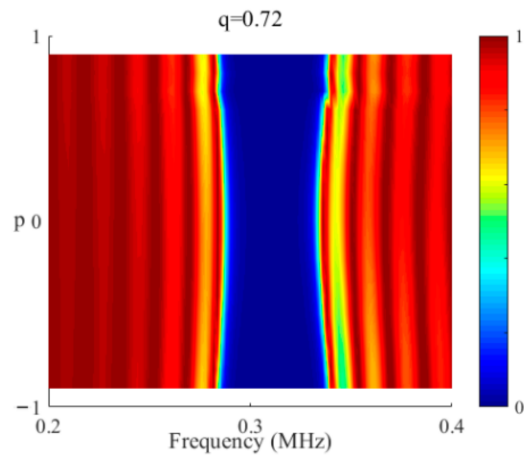

(e)

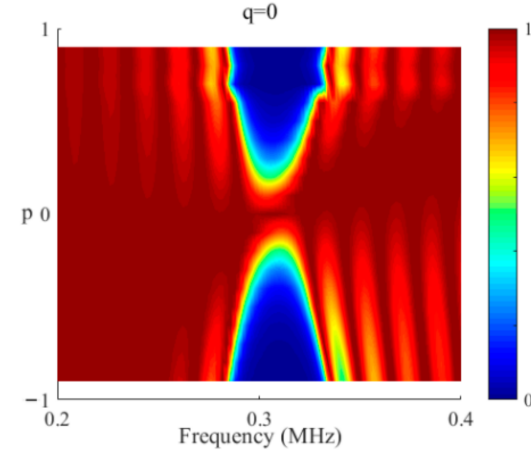

(c)

Figure 3. The TS through the PC plates with different parameters $p$ and $q$. (a) $q=-0.72$, (b) $q=-0.36$, (c) $q=0$, (d) $q=0.36$, (e) $q=0.72$. The band structures with negative $q$ are the same as the band structures with the positive " $q$ ", which leads to the same TS.

The RP of a PC plate, which is surrounded by a perfectly matched layer and a rigid boundary, is also studied. Regarding the effective Hamiltonian, the non-trivial topology of the synthetic WP results in the formation of an eddy current-like RP structure in the parameter space, where the number of quantized phase windings is identical with the corresponding Weyl charge [2]. The distributions of RP are shown Figure 3a, where the frequency $0.306 \mathrm{MHz}$ of the Weyl point between band 1 and band 2 is used. The phase distribution in the $p-q$ space clearly shows a vortex. The reflection simulation proved the non-trivial phase distribution.

Robust RP singularity is a new characteristic of topologically stable PC plates. At the same time, the vortex phase structure ensures the presence of interface states, and each WP is associated with an interface state. In Figure $4 b$, the energy density spectrum of the phononic crystal plate with seventeen periods at $(p, q)=(0.35,0.45)$, normalized by the flat panel is calculated. There is a significant peak value appearing at $0.305 \mathrm{MHz}$, which is in line with the interface transition frequency in Figure $4 a$, which verifies the topologically non-trivial interface state.

To visualize the presence of topological interface states, the displacement distribution of the PC plate at a Weyl frequency of $0.305 \mathrm{MHz}$ with $(p, q)=(0.35,0.45)$ is plotted in Figure 5 . We can visually and clearly see that the wave motion is concentrated between the rigid boundary and the PC plate, which is a typical property of the topological interface state. This strongly confined property has potential applications in designing elastic sensors and harvesting vibration energy. In order to quantitatively show the large differences in 
displacement amplitude, the distribution of displacement amplitude along the $\mathrm{x}$-direction is also given in Figure 5.

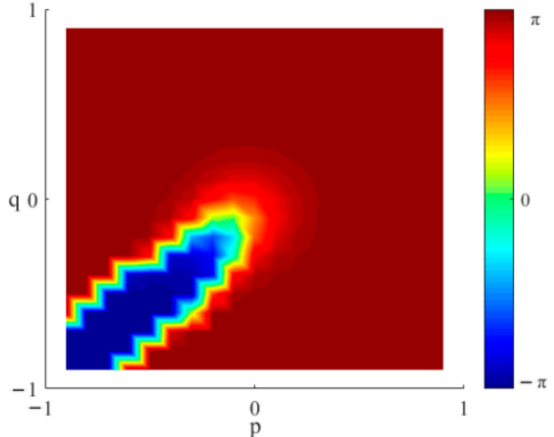

(a)

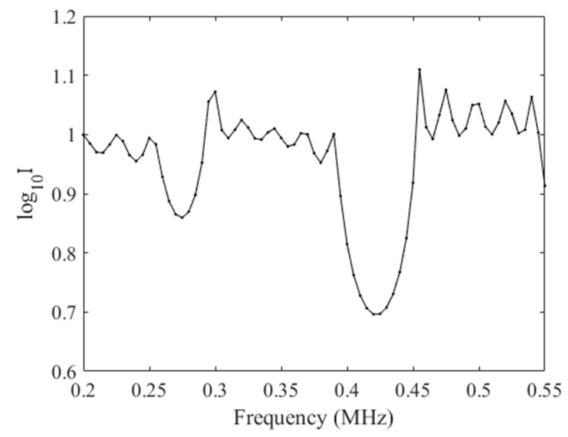

(b)

Figure 4. RP singularity and subsequent topology interface state. (a) The RPs of PC plates with different parameters are calculated at a Weyl frequency of $0.305 \mathrm{MHz}$. (b) The energy density spectrum normalized by the flat panel at $(p, q)=(0.35,0.45)$.

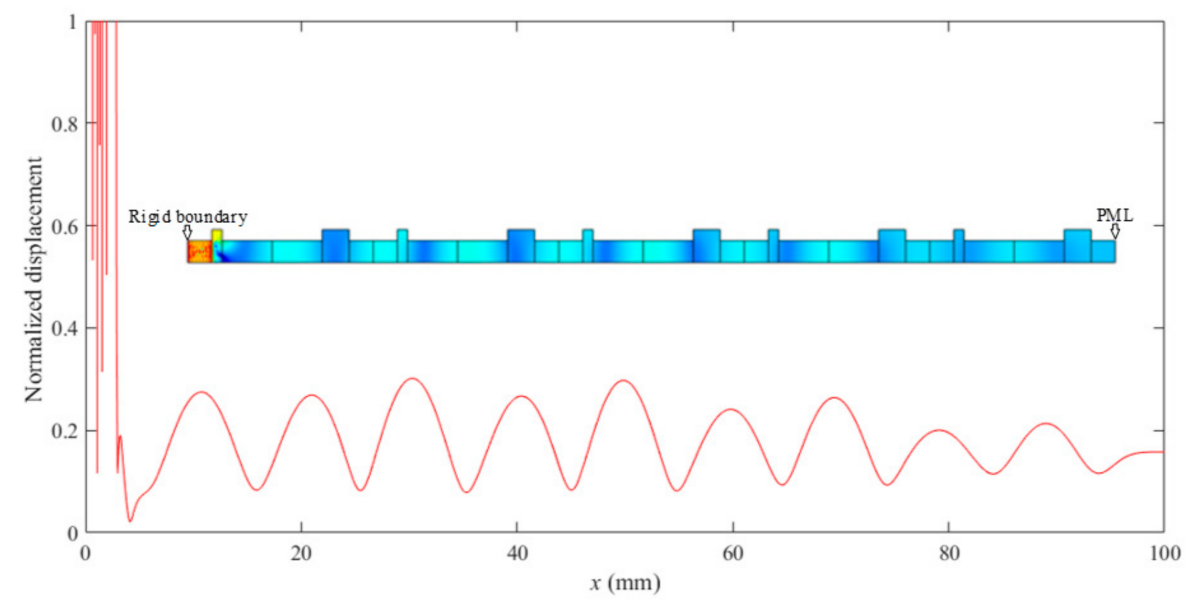

Figure 5. The displacement distribution of the PC plate at a Weyl frequency of $0.305 \mathrm{MHz}$ with $(p, q)=(0.35,0.45)$

Finally, the TR for detecting the WP are conceived as follows. The sample can be made by laser cutting technique. The ultrasonic experiments can be used to obtain the TR. The detailed test procedure is as follows. First, a PZT transducer bonded on the upper surface is driven by a short duration pulse generated by the generator at the left side. Second, a fiber Bragg grating sensor bonded on the upper surface is used to receive wave signals at the right side. Third, the collected signals are processed in the oscilloscope and then the TR can be obtained.

\section{Discussion}

The synthetic WPs of the SH guided waves in 1D PC plates are investigated. Here a virtual 3D space is formed by the Bloch wave vector plus the two additional parameters. Base on the zone folding mechanism, the WPs can be obtained. Furthermore, we also obtain the WP by changing one structural parameter. In order to confirm the WP, the conic intersection in the bulk dispersions is also presented in $p-q$ space. To further confirm the synthesized WPs, the frequency-dependent TS through 1D PC plates with different structural parameters are investigated. Besides, the RP of a semi-infinite SC with a rigid boundary is also studied, and a vortex as the signature of Weyl physics is found in the phase distribution. The vortex phase structure ensures the presence of interface states, and 
the interface state is also shown in the TS. This study may facilitate several operational applications. For example, the interface states can enhance the elastic energy and thus can be combined into directional elastic antenna structures, providing a technical means for communication and detection.

Author Contributions: Conceptualization, H.Z.; Investigation, H.Z.; Methodology, J.L.; Software, S.Z.; Writing—original draft, H.Z.; Writing—review \& editing, B.L. All authors have read and agreed to the published version of the manuscript.

Funding: This research work is supported by the National Natural Science Foundation of China (NSFC) (Grant No. 51575288 Grant No. 11874034) and the Taishan Scholar Program of Shandong (Grant No. ts201712054). The authors gratefully acknowledge the supporting agency.

Data Availability Statement: All data that support the findings of this study are included within the article.

Conflicts of Interest: The authors declare no conflict of interest. The funders had no role in the design of the study; in the collection, analyses, or interpretation of data; in the writing of the manuscript, or in the decision to publish the results.

\section{References}

1. Fan, X.; Qiu, C.; Shen, Y.; He, H.; Xiao, M.; Ke, M.; Liu, Z. Probing Weyl Physics with One-Dimensional Sonic Crystals. Phys. Rev. Lett. 2019, 122, 1079-7114. [CrossRef] [PubMed]

2. Wang, Q.; Xiao, M.; Liu, H.; Zhu, S.; Chan, C.T. Optical Interface States Protected by Synthetic Weyl Points. Phys. Rev. X 2017, 7, 031032. [CrossRef]

3. Zhang, H.; Liu, B.; Zhang, X.; Wu, Q.; Wang, X. Zone folding induced tunable topological interface states in one-dimensional phononic crystal plates. Phys. Lett. A 2019, 383, 2797-2801. [CrossRef]

4. Burkov, A.A.; Balents, L. Weyl Semimetal in a Topological Insulator Multilayer. Phys. Rev. Lett. 2011, 107, 127205. [CrossRef] [PubMed]

5. Huang, X.; Zhao, L.; Long, Y.; Wang, P.; Chen, D.; Yang, Z.; Liang, H.; Xue, M.; Weng, H.; Fang, Z.; et al. Observation of the Chiral-Anomaly-Induced Negative Magnetoresistance in 3D Weyl Semimetal TaAs. Phys. Rev. X 2015, 5, 031023. [CrossRef]

6. Xu, G.; Weng, H.; Wang, Z.; Dai, X.; Fang, Z. Chern Semimetal and the Quantized Anomalous Hall Effect in $\mathrm{HgCr}_{2} \mathrm{Se}_{4}$. Phys. Rev. Lett. 2011, 107, 186806. [CrossRef] [PubMed]

7. Xu, S.Y.; Belopolski, I.; Alidoust, N.; Neupane, M.; Bian, G.; Zhang, C.; Sankar, R.; Chang, G.; Yuan, Z.; Lee, C.C.; et al. Discovery of a Weyl fermion semimetal and topological Fermi arcs. Science 2015, 349, 613-617. [CrossRef] [PubMed]

8. Lv, B.Q.; Weng, H.; Fu, B.B.; Wang, X.P.; Miao, H.; Ma, J.; Richard, P.; Huang, X.C.; Zhao, L.X.; Chen, G.F.; et al. Experimental Discovery of Weyl Semimetal TaAs. Phys. Rev. X 2015, 5, 031013. [CrossRef]

9. Wan, X.; Turner, A.M.; Vishwanath, A.; Savrasov, S.Y. Topological semimetal and Fermi-arc surface states in the electronic structure of pyrochlore iridates. Phys. Rev. B 2011, 83, 205101-205109. [CrossRef]

10. Ge, H.; Ni, X.; Tian, Y.; Gupta, S.K.; Lu, M.H.; Lin, X.; Huang, W.D.; Chan, C.T.; Chen, Y.F. Experimental Observation of Acoustic Weyl Points and Topological Surface States. Phys. Rev. Appl. 2018, 10, 014017. [CrossRef]

11. He, H.; Qiu, C.; Ye, L.; Cai, X.; Fan, X.; Ke, M.; Zhang, F.; Liu, Z. Topological negative refraction of surface acoustic waves in a Weyl phononic crystal. Nature 2018, 560, 61-64. [CrossRef] [PubMed]

12. Li, F.; Huang, X.; Lu, J.; Ma, J.; Liu, Z. Weyl points and Fermi arcs in a chiral phononic crystal. Nat. Phys. 2017, 14, 30-34. [CrossRef]

13. Xiao, M.; Chen, W.J.; He, W.Y.; Chan, C.T. Synthetic gauge flux and Weyl points in acoustic systems. Nat. Phys. 2015, 11, 920-924. [CrossRef]

14. Yang, Z.; Zhang, B. Acoustic Type-II Weyl Nodes from Stacking Dimerized Chains. Phys. Rev. Lett. 2016, 117, 224301. [CrossRef] [PubMed]

15. Xiao, M.; Zhang, Z.Q.; Chan, C.T. Surface Impedance and Bulk Band Geometric Phases in One-Dimensional Systems. Phys. Rev. $X$ 2014, 4, 130-136. [CrossRef]

16. Zhu, W.; Ding, Y.Q.; Ren, J.; Sun, Y.; Li, Y.; Jiang, H.; Chen, H. Zak phase and band inversion in dimerized one-dimensional locally resonant metamaterials. Phys. Rev. B 2018, 97, 195307. [CrossRef]

17. Downing, C.A.; Weick, G. Topological collective plasmons in bipartite chains of metallic nanoparticles. Phys. Rev. 2017, 95, 125426. [CrossRef]

18. Feng, L.Y.; Huang, H.B.; Zhang, J.C.; Xie, X.P.; Chen, J.J. Reconfigurable topological phononic crystal slabs. Phys. Lett. A 2018, 382, 2880-2885. [CrossRef]

19. Huang, H.; Chen, J.J.; Huo, S. Simultaneous topological Bragg and locally resonant edge modes of shear horizontal guided wave in one-dimensional structure. J. Phys. D Appl. Phys. 2017, 50, 275102. [CrossRef] 
20. Yan, M.; Lu, J.; Li, F.; Deng, W.; Huang, X.; Ma, J.; Liu, Z. On-chip valley topological materials for elastic wave manipulation. Nat. Mater. 2018, 17, 993-998. [CrossRef]

21. Yin, J.; Li, L.; Zhang, H.; Li, X.; Gao, A.; Zhu, H. A prediction-based coordination caching scheme for content centric networking. In Proceedings of the 2018 27th Wireless and Optical Communication Conference (WOCC), Hualien, Taiwan, 30 April-1 May 2018.

22. Chen, J.; Huo, S.; Geng, Z.G.; Huang, H.; Zhu, X.F. Topological valley transport of plate-mode waves in a homogenous thin plate with periodic stubbed surface. AIP Adv. 2017, 7, 115215. [CrossRef]

23. Kim, I.; Iwamoto, S.; Arakawa, Y. Topologically protected elastic waves in one-dimensional phononic crystals of continuous media. Appl. Phys. Express 2018, 11, 017201. [CrossRef]

24. Pal, R.K.; Ruzzene, M. Edge waves in plates with resonators: An elastic analogue of the quantum valley Hall effect. New J. Phys. 2016, 19, 025001. [CrossRef]

25. Roushan, P.; Neill, C.; Chen, Y.; Kolodrubetz, M.; Quintana, C.; Leung, N.; Fang, M.; Barends, R.; Campbell, B.; Chen, Z.; et al. Observation of topological transitions in interacting quantum circuits. Nature 2014, 515, 241-244. [CrossRef]

26. Schroer, M.D.; Kolodrubetz, M.H.; Kindel, W.F.; Sandberg, M.; Gao, J.; Vissers, M.R.; Pappas, D.P.; Polkovnikov, A.; Lehnert, K.W. Measuring a Topological Transition in an Artificial Spin-1/2System. Phys. Rev. Lett. 2014, 113, 50402. [CrossRef] [PubMed]

27. Riwar, R.P.; Houzet, M.; Meyer, J.S.; Nazarov, Y.V. Multi-terminal Josephson junctions as topological matter. Nat. Commun. 2016, 7, 11167. [CrossRef] [PubMed]

28. Fleury, R.; Sounas, D.L.; Sieck, C.F.; Haberman, M.R.; Alù, A. Sound Isolation and Giant Linear Nonreciprocity in a Compact Acoustic Circulator. Science 2014, 343, 516-519. [CrossRef]

29. Yang, Z.; Gao, F.; Shi, X.; Lin, X.; Gao, Z.; Chong, Y.; Zhang, B. Topological Acoustics. Phys. Rev. Lett. 2015, 114, 114301. [CrossRef] [PubMed]

30. Soluyanov, A.A.; Gresch, D.; Wang, Z.; Wu, Q.; Troyer, M.; Dai, X.; Bernevig, B.A. Type-II Weyl semimetals. Nature 2015, 527, 495-498. [CrossRef]

31. Lin, Q.; Xiao, M.; Yuan, L.; Fan, S. Photonic Weyl point in a two-dimensional resonator lattice with a synthetic frequency dimension. Nat. Commun. 2016, 7, 13731. [CrossRef] 